\title{
Extraction of QRS Fiducial Points from the ECG Using Adaptive Mathematical Morphology
}

\author{
Sasan Yazdani ${ }^{1, *}$, Jean-Marc Vesin ${ }^{1}$ \\ ${ }^{1}$ Department of Electrical Engineering, Swiss Federal Institute of Technology (EPFL), CH-1015 Lausanne, Switzerland \\ ${ }^{*}$ Corresponding author:sasan.yazdani@epfl.ch. Author:jean-marc.vesin@epfl.ch
}

\begin{abstract}
QRS complex detection in the electrocardiogram (ECG) has been extensively investigated over the last two decades. Still, some issues remain pending due to the diversity of QRS complex shapes and various perturbations, notably baseline drift. This is especially true for ECG signals acquired using wearable devices. Our study aims at extracting QRS complexes and their fiducial points using Mathematical Morphology (MM) with an adaptive structuring element, on a beat-to-beat basis. The structuring element is updated based on the characteristics of the previously detected QRS complexes for a more robust and precise detection. The MIT-BIH arrhythmia and Physionet QT databases were respectively used for assessing the detection performance of R-waves and other fiducial points. Furthermore, the proposed method was evaluated on a wearable-device dataset of ECGs during vigorous exercises. Results show comparable or better performance than the state-of-the-art with a $99.87 \%$ sensitivity and $0.22 \%$ detection error rate for the MIT-BIH arrhythmia database. Efficient extraction of QRS fiducial points was achieved against the Physionet QT database. On the wearable-device dataset, an improvement of more than $10 \%$ in QRS complex detection rate compared to classic approaches was obtained.
\end{abstract}

Keywords. QRS complex extraction; QRS fiducial point extraction; Wearable ECG; Adaptive Mathematical Morphology;

\section{INTRODUCTION}

Electrical activity propagated through the myocardium provides valuable information about the state of the heart. This electrical activity is reflected on the body surface and can be captured using the electrocardiograph. The electrocardiogram (ECG) comprises different electrical waveforms, each representing either depolarization or repolarization of different muscles in the heart. Among these waveforms, the QRS complex, corresponding to the ventricular contraction is the most prominent. The shape of this complex as well as the time of its appearance provides significant information for arrhythmia [1], and heart rate variability analysis [2]. Due to its peaky shape and the fact that other waveforms can be small, or in some cases not even present in the ECG, the QRS complex plays a fundamental role in the automatic detection of heartbeats.

However, the detection of QRS complexes in the ECG is not always an easy task due to the physiological variability of the QRS and more importantly, the presence of perturbations caused by different sources such as power-line interference, muscle activity and baseline drift. Many QRS detection approaches have been proposed in the literature. Thorough reviews and comparisons of numerous traditional methods can be found in [3] and [4]. Generally, approaches consider two stages in QRS complex detection, namely the filtering stage and the decision 5 tage. In $\square$ most methods, the ECG is first preprocessed by a low-pass filter to remove the noise introduced by the electrocardiograph usually at 50 or $60 \mathrm{~Hz}$. Some approaches employ a band-pass filter with cutoff frequencies at about 10 and $25 \mathrm{~Hz}$ not only to suppress the acquisition noise, but also other waveforms such as the P-wave, T-wave and the baseline drift.

Subsequently, in the decision stage, a feature signal is extracted from the filter output and compared with some heuristically chosen thresholds to determine whether a QRS complex is taking place at a certain point in the ECG. Among these approaches, Pan and Tompkins [5] proposed a derivative-based approach taking advantage of the steep slope characteristic of the QRS complex in the detection phase. They used simple difference equations alongside a band pass filter to point out QRS complexes. Li et al. [6] applied a wavelet transform (WT) approach to the ECG and found that R-peaks can be picked out from perturbations such as biseline drift and other waveforms in the ECG if there are concurrent local modulus maxima at different WT scales. Trahanias [7] used mathematical morphology (MM) operators on the ECG both in the noise removal and detection phases, each phase Zising a different fixed structuring element (SE). More recently, Zhang and Lian [8] proposed a three-stage MM approach. They studied how SEs with different lengths and slopes affect the output of the MM sperators. Yet, at each filtering stage, a fixed SE was employed by the MM operators. Finally the extracted feature signal was compared with a fixed or adaptive threshold to detect R-peaks.

To the best of our knowledge, all QRS detection approaches use some thresholds to detect these complexes in the ECG. These thresholds comprise physiological constraints, e.g. the time interval between two beats cannot be smaller than 250 milliseconds, and arbitrary thresholds, e.g. comparing the feature signal to a specific value to decrease the false detection rate. The former are always valid and can be useful for detection but the latter are based on the data at hand, can be hard to adapt when dealing with several subjects, or may need adjustment in different acquisition scenarios. In this paper we aim to present a method that takes advantage of physiological constraints and includes empirically selected arbitrary thresholds. 


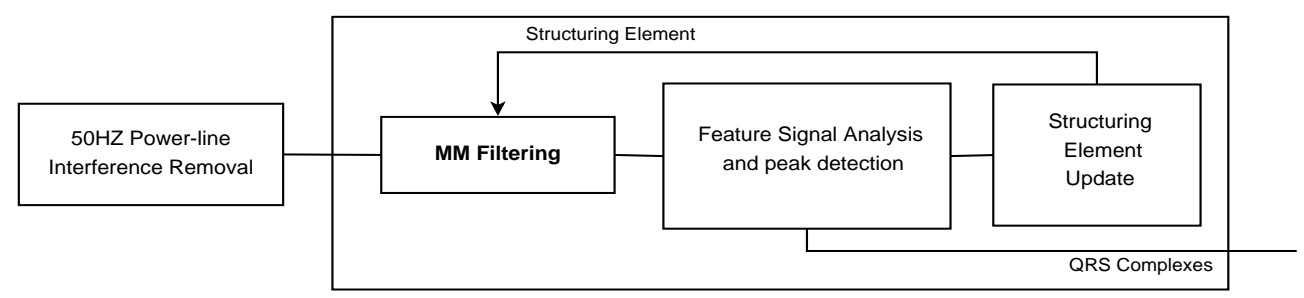

Fig. 1. Block Diagram of AMM.

This paper contains a more comprehensive study of an MM approach with an adaptive SE, presented first in the conference CinC 2014 [9]. The proposed method is expected to be robust against baseline drift and other perturbations with low computational costs and good performance. The adaptive SE uses the shape of the QRS complex waveform not only to extract the R-waves, but also to estimate the location of other fiducial points in the QRS complex.

The rest of the paper is organized as follows. Section $\square$ focuses on a brief introduction on MM. The proposed approach and its implementation are explained in section B. Section 1 provides the description of the evaluation databases used in this study. In Section [, the experimental results are presented and the quality of the QRS complex detection is validated. Finally in Section 6, the main conclusions of this work are drawn.

\section{Mathematical Morphology (MM)}

MM is a methodology proposed to extract topological information based on the analysis of geometrical structures. MM was first introduced for binary images with strong set-theoretic concepts, designed to extract useful information in images regarding shape and size [10]. Operators in MM nonlinearly transform the signal of interest using another signal called the structuring element (SE). The outcome of an MM operator depends on the shape and the length of the SE.

Essentially, MM is based on two elementary operators named dilation and erosion. Combining dilation and erosion leads to additional operators such as opening, closing, top-hat and bottom-hat, definition of which are listed as follows:

$$
\begin{gathered}
\text { Dilation } \oplus:(f \oplus g)(n)=\max _{i}\{f(n-i)+g(i)\} \\
\text { Erosion } \ominus:(f \ominus g)(n)=\min _{i}\{f(n+i)-g(i)\} \\
\text { Opening } \circ: f \circ g=(f \ominus g) \oplus g \\
\text { Closing }: f \bullet g=(f \oplus g) \ominus g \\
\text { Top_Hat }: \text { THat }(f)=f-f \circ g \\
\text { Bottom_Hat }: \text { BHat }(f)=f-f \bullet g
\end{gathered}
$$

Where $g$ represents the SE with a length of $L, i$ indicating the $i$ th element of the SE. $f$ is the signal with a length of $N$ to which the MM operator is applied and $n$ represents the $n$th sample of $f$. These operators are easy to compute and simply defined. Therefore, they constitute a suitable option in cases where computation load plays an important role such as body-area networks.

In biomedical signal processing and more specifically ECG QRS complex detection, MM can be very useful due to the distinct shape of the QRS complex. By employing a peaky structure in MM operations, a filter can be designed which makes QRS complexes more prominent and suppresses other waveforms, the desired effect sought in QRS complex detection.

\section{Proposed method}

In this paper we propose an MM approach with an adaptive SE (AMM). This adaptation takes advantage of the topological features of subject heartbeats and uses them for a more robust and precise detection. AMM can be considered as a 4-step algorithm, a block diagram of which is shown in Fig. 1 .

Before the detection of QRS complexes in the ECG is carried out, the signal must be conditioned by removing different potential perturbations. Power-line interference, high frequency muscle activity and low-frequency baseline drift are among the most dominant perturbations. The proposed method uses a low-pass filter with a cutoff frequency at $50 \mathrm{~Hz}$, mainly to remove the power-line interference. 


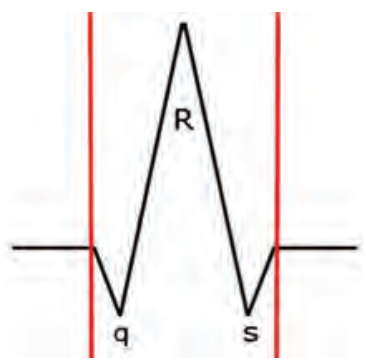

(a)

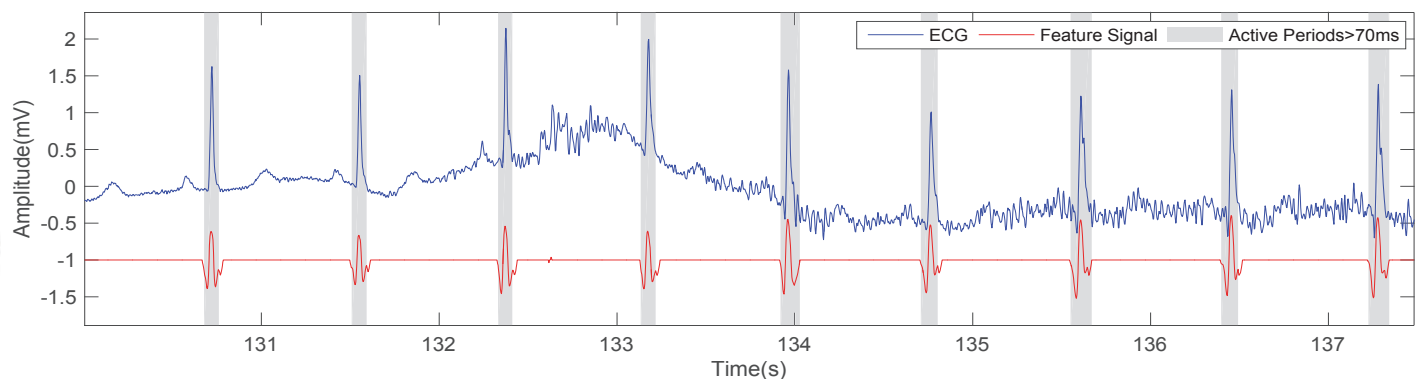

(b)

Fig. 2. (a) Synthesized QRS structuring element. The vertical lines before the Q-point and after the S-point respectively represent the onset and offset of the structuring element. The iso-electric lines are added to this figure for demonstration purposes. (b) Extracted feature signal, i.e. the output of the MM filtering phase. For the purpose of demonstration, the signal length of 8 seconds is chosen. Also, a $-1 \mathrm{mv}$ DC value is added to the extracted feature signal.

\subsection{Filtering}

As mentioned in section 2, MM operators use an SE to manipulate the signal. However, there is no available information about the shape and magnitude of the QRS complexes of a specific subject, before detecting heartbeats in the ECG. Therefore, a synthesized QRS-like SE is necessary for the initialization of AMM. This SE is synthesized using a priori knowledge of a general QRS complex. Like a typical heartbeat complex, this SE comprises five fiducial points namely, R-peak, Q-point, S-point together with onset and offset, equally spaced in time in the synthesized element, as shown in Fig. D-a. The duration of the element is 90 milliseconds, which represents an average normal QRS duration [II]. However, the magnitude of the SE is subject dependent and cannot be determined using a priori knowledge. Therefore, this value should be determined from the ECG at hand. To initialize AMM, the magnitude of the SE is considered as the difference between the maximum and minimum of the first 2-seconds of the ECG.

Using the synthesized SE, the average of top-hat and bottom-hat is calculated on a one-second window of the ECG. This

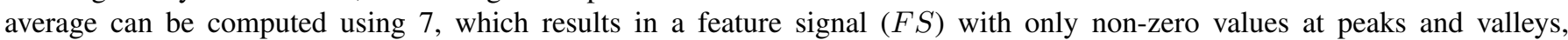
mostly corresponding to QRS complexes.

$$
F S=f-\frac{f \circ g+f \bullet g}{2}
$$

The top- and bottom-hat operators respectively give rise to peaks and valleys at their output. The average of these operators extracts the peaks and valleys from the original signal, i.e. $f$, and discards other activities.

\subsection{Feature Signal Analysis and Peak Detection}

After the MM filtering phase of AMM, the extracted feature signal is scrutinized for QRS complex detection. In this phase, first, non-zero segments of the feature signal, defined as segments in the feature signal without three consecutive zero values, are extracted. Then, non-zero segments (referred to as active periods) with durations longer than 70ms are considered as QRS candidates. This 70ms threshold on the minimum duration of a QRS candidate is set based on the physiological constraint of minimum possible QRS complex duration observed in patients with extreme heart conditions [12] [13]].

Fig. D-b illustrates the feature analysis phase of AMM on an 8-second window of tape 101 from the MIT/BIH arrhythmia database. In this figure, a low-amplitude active period with a duration of $8 \mathrm{~ms}$ takes place around second 133 of the feature signal extracted from the tape, and therefore it is discarded as a non-candidate section. The highlighted sections of this figure represent the extracted QRS candidates.

Subsequently, active periods with duration longer than $70 \mathrm{~ms}$ are further processed as follows:

1) QRS-onset and QRS-offset are temporarily considered respectively as the start and end of the active period.

2) R-peak defined as the most significant peak, and the polarity of R-peak are extracted from the active period. Schematics of this sub algorithm is illustrated in fig. B].

3) The minimum (maximum) between the onset and R-peak is considered as the Q-point of the QRS complex, in case R-peak has a positive (negative) polarity.

4) The minimum (maximum) between R-peak and the offset point is considered as the S-point of the complex, in case R-peak has a positive (negative) polarity.

5) QRS-onset (QRS-offset) is shifted to a maximum or minimum of the features signal, if it exists between the provisional onset (offset) and the Q-point (S-point).

In Fig. $\mathbb{Z}$-b, highlighted sections show the extracted onset-offset periods for every viable QRS-candidate, based on steps 5 and 6. 


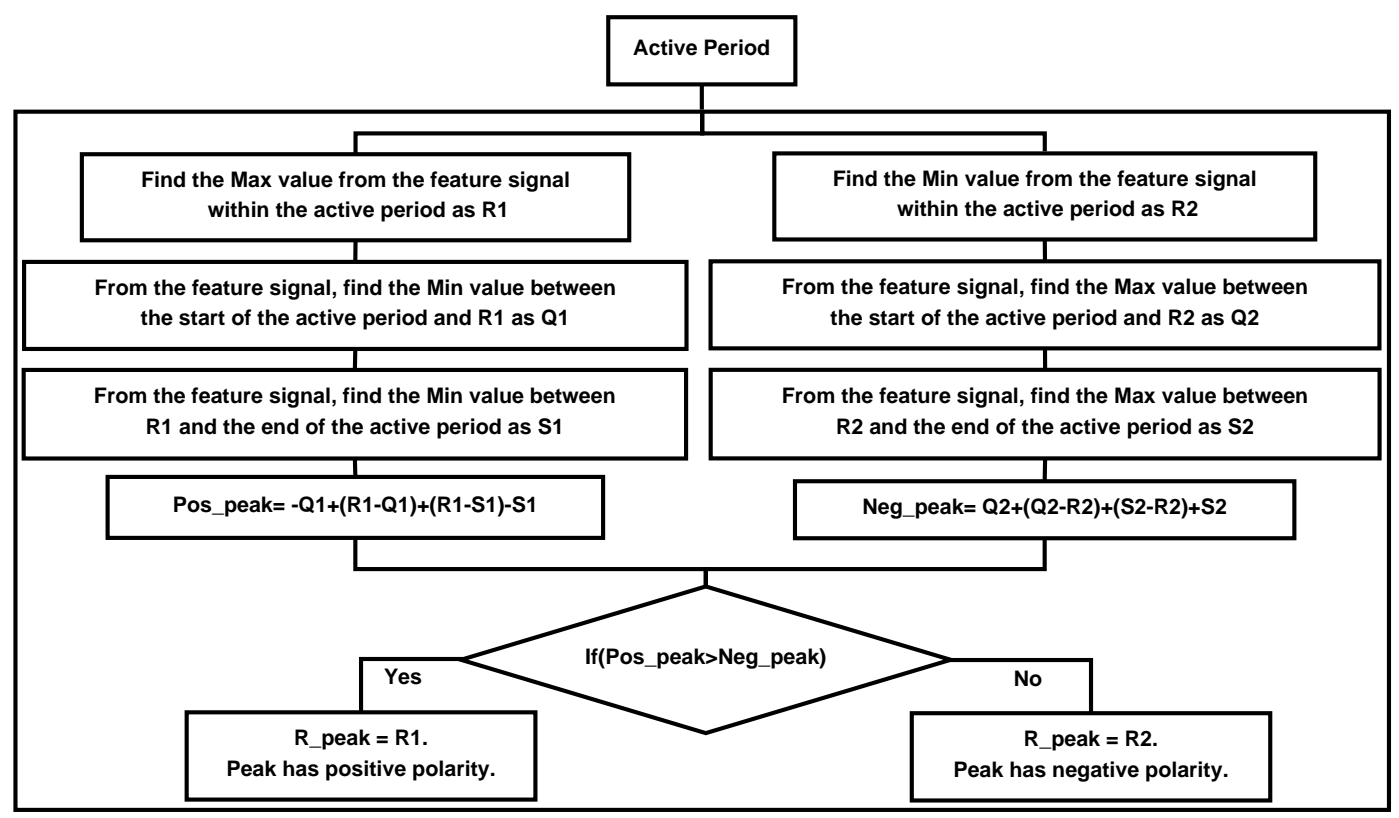

Fig. 3. $A M M$ approach on detecting the polarity of the QRS complex.

\subsection{Structuring Element Update}

After the detection of a QRS complex, the update phase of AMM is invoked in order to adapt the SE so that it best represents the current QRS morphology of the subject. In other words, this phase extracts features such as shape, length and magnitude of the newly detected complex and uses them to update the SE to enhance QRS detection. More specifically, for each fiducial point, first its amplitude and relative distance with respect to the QRS-onset are extracted from the feature signal. Then, using a learning coefficient, location indices and amplitude values are updated based on the extracted topological features from the newly detected QRS and the ones from the synthesized QRS complexes, as shown in the following equations:

$$
\begin{gathered}
\text { NewLoc }=(1-\alpha) \times \text { Curr_Loc }+\alpha \times \text { ExtractedLoc } \\
\text { NewAmp }=(1-\alpha) \times \text { Curr_Amp }+\alpha \times \text { ExtractedAmp }
\end{gathered}
$$

In these equations, $\alpha$ represents the learning coefficient. For each fiducial point, Curr_Loc is extracted from the current $\mathrm{SE}$, calculated as its time distance with regard to the QRS onset. ExtractedLoc, is extracted in the same manner for each fiducial point, using the feature signal. Additionally, Curr_amp and ExtractedAmp represent the amplitude of each fiducial point respectively in the current SE and the feature signal.

Once NewLoc, and NewAmp values have been calculated using the extracted onset, offset, Q-point, S-point and R-peak, the SE is reconstructed by means of linear interpolation. The updated SE is later used in the MM filtering phase of AMM in

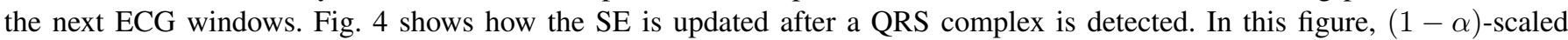
Current SE and $\alpha$-scaled Extracted SE illustrate simultaneous changes on amplitude and location, respectively on the current structuring element and the extracted SE. The structuring element update of the AMM ensures that SE always has a positive polarity. When inverted QRS polarities are detected, the update on SE is performed on the negated amplitude values of the fiducial points in order to avoid zeroing of the update SE.

Generally, AMM processes the ECG on a non-overlapping second-by-second basis. Nevertheless, sometimes only a part of a QRS complex is accessible through the one-second sliding window. In these cases where the feature signal ends while a non-zero period is not completely extracted, the window is slided to the start of the final non-zero period instead of the start of a new one-second window.

Ideally, after the detection of a complex, the updated MM filter should be applied to the signal from the last extracted offset point. However, our observations have shown that the QRS complex morphology variations are usually small when multiple active periods are detected in the one-second feature signal, i.e. in case of tachycardia or when the instantaneous heart rate is higher than 120 beats per minute. Therefore, in order to avoid computational overhead, the MM filter is applied only once to each window of the ECG signal. Still, the update on the SE takes place after the detection of each heartbeat so that it represents the current QRS morphology of the subject for the future ECG windows.

On the other hand, if the subject has an instantaneous heart rate lower than 60 beats per minute, e.g. when dealing with bradycardia, mobitz or asystole, it is possible that no active periods are found in the feature signal. In these cases the MM filtering is applied to the next window of the ECG. 


\subsection{Parameter Update}

Due to perturbations, small activity periods of the non-zero feature signal [longer than 70ms] may be spotted at positions where QRS complexes do not occur. Based on our observations, these non-zero activities mostly take place right before or after an actual heartbeat due to P-waves or T-waves that take place closer than usual to the QRS complexes. To address this issue, an additional feature is extracted for each active period in the feature signal called PeakActivity (PA). This feature is defined as follows.

$$
P A_{Q R S-\text { candidate }}=\sum_{i \in \text { Nonzero-Activity }}|F S(i)|
$$

where $F S$ is the extracted feature signal. Since the feature signal artifacts are usually located right after or before an actual QRS complex, they can be effortlessly removed since it is physiologically unlikely to have two consecutive heartbeats within $250 \mathrm{~ms}$ [14]. Therefore, if two QRS consecutive candidates take place in an interval smaller than $250 \mathrm{~ms}$, the candidate with the bigger PA value is selected.

Another issue is that a fixed learning coefficient in Eq. $8-9$ might lead to active periods in the feature signal that cannot precisely estimate the onset and offset points of the corresponding QRS complex. To alleviate this issue, after the detection of a complex, the newly extracted $P A$ feature is compared with that of the previous heartbeat and as a result, the learning coefficient $\alpha$ is updated using Eq. W.

$$
\alpha= \begin{cases}\alpha-\delta & P A_{\text {Extracted }}>P A_{\text {Previous }} \times t h_{\text {high }} \\ \alpha+\delta & P A_{\text {Extracted }}<P A_{\text {Previous }} \times t h_{\text {low }} \\ 0.3 & \text { otherwise }\end{cases}
$$

where $\delta$ is the step size with which the learning coefficent is adjusted. $t h_{h i g h}$ and $t h_{l o w}$ respectively define the lower and higher $P A$ thresholds. $P A_{\text {Extracted }}$ and PA Previous respectively represent the extracted and previous PeakActivity.

Our studies have shown that a default $\alpha$ value of 0.3 and a $\delta$ of 0.05 are optimal for the learning coefficient. Also, $t h_{l o w}$ and th high were chosen as 0.9 and 1.1 respectively (see the results and discussion section). Since the initializing SE does not fully represent the QRS complex morphology of the subject, $\alpha$ is set to 0.9 at the beginning of the algorithm. After detection of the second QRS complex, the PeakActivity feature of the newly detected QRS complex is compared to that of the previous heartbeat and $\alpha$ is updated using Eq. 피.

This $P A$ feedback plays an important role when sudden changes happen in the ECG, such as baseline drift, or when respiration highly modulates the amplitude of the R-waves. When there is a decrease larger than 10 percent in $P A$, the SE should be updated to look more like the newly found QRS since a sudden change takes place in the ECG. This phenomenon is usually observed when baseline drift is present in the ECG. On the other hand, a sudden increase in $P A$ indicates that the $\mathrm{SE}$ should be less affected to avoid miss detection of future beats. Thus, the decrease in $\alpha$ is needed when an irregular beat with higher $P A$ occurs in the ECG, such as premature ventricular contraction. Results show not only this adaptation improves heartbeat detection but also provides accurate positions of the $\mathrm{Q}, \mathrm{S}$, onset and offset points.

The last phase of the algorithm, i.e. the SE and parameter update, makes AMM flexible and robust again purterbations. Fig. 5 illustrates the performance of AMM on a part of tape number 108 of the MIT/BIH arrhythmia database. This tape is among the most difficult ones since it is polluted with high amplitude perturbations.

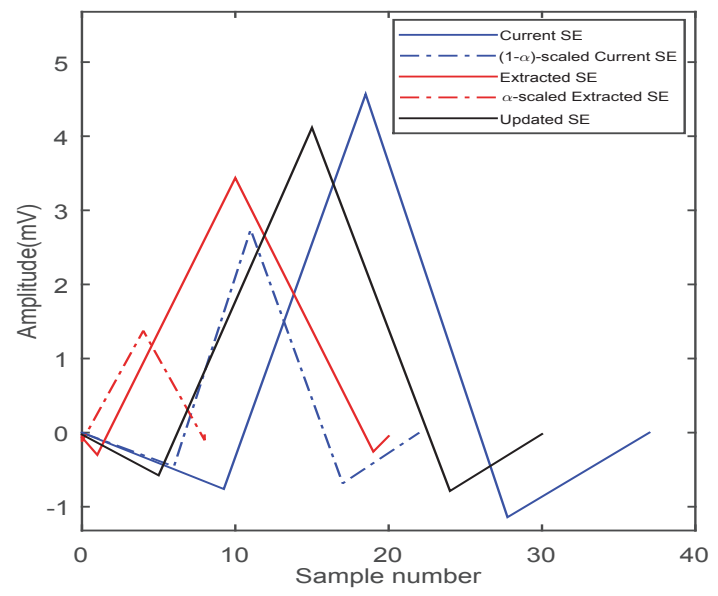

Fig. 4. The Structuring update phase of AMM. This update takes place after detection of a QRS complex for a better and more precise detection of R-waves and other fiducial points in the complex. $\alpha$ was considered as 0.4 for demonstration. 


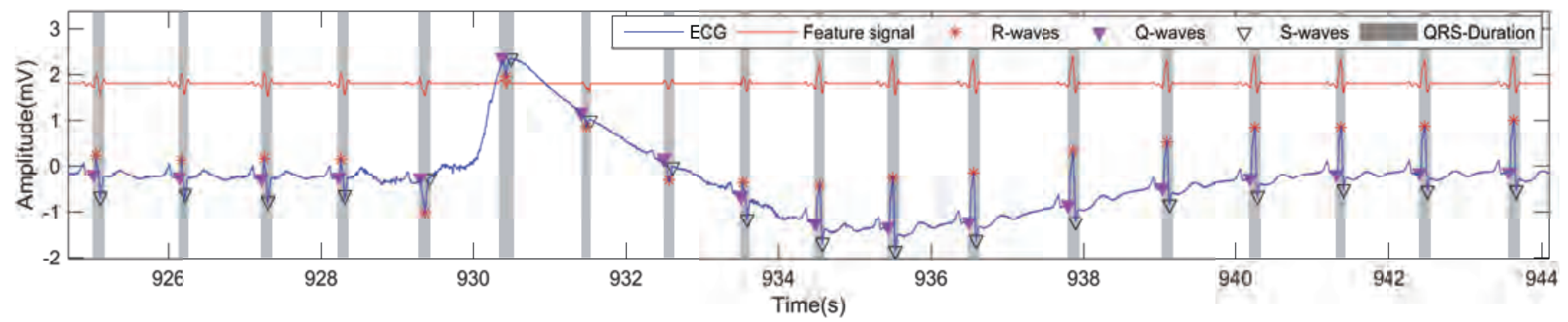

Fig. 5. Illustration of AMM performance on tape no. 108 from the MIT/BIH arrhythmia. A DC value of $1.8 \mathrm{mv}$ is added to the feature signal.

\section{Evaluation Databases}

In order to find the optimal parameters for AMM as well as to evaluate the performance of the proposed method, the MIT/BIH arrhythmia [15] and Physionet QT [16] databases were used for the detection of QRS and other fiducial points. The MIT/BIH arrhythmia database consists of 48 half hour two-channel ECGs with $360 \mathrm{~Hz}$ sampling frequency and 11-bit resolution on a range of 10-mv. For each recording in the MIT/BIH arrhythmia database, heartbeats were independently annotated by two or more cardiologists and then disagreements were resolved to provide a reference annotation file. For this databases, the QRS detection performance was evaluated on the first ECG channel (modified lead II), as AMM works on a single lead basis.

The QT database, designed for waveform boundary evaluation, consists of 105 15-minute two-channel ECGs with a variety of QRS morphologies sampled at $250 \mathrm{~Hz}$. The QT database was used in order to evaluate the delineation performance of AMM. In this database, for each record at least 30 beats (from 30 to more than 100 beats) were manually annotated. The records for the QT database were annotated by two cardiologist, who provided annotations by examining both ECG channels. One expert annotated all tapes in the database while the other only provided annotations for 11 records. In this study, for a precise evaluation on boundary and fiducial point detection, only the manually annotated waveforms, totaling 3622 beats, were considered in the experiments. In other words, we used the annotations provided by the cardiologist who examined all records of the QT database. In order to have a fair comparison with the cardiologist annotations, both channels were processed separately by AMM and for each point the channel with less error was selected.

Finally, to evaluate AMM performance on wearable acquisition platforms, we used an energy expenditure database recorded from smart shirts, developed at Swiss Center for Electronics and Microtechnology (CSEM). In this database, ECGs were acquired at $250 \mathrm{~Hz}$ from chest-located dry electrodes, simultaneously over 13 healthy male subjects, in laboratory settings. The distribution of anthropometric parameters of the subjects is shown in Table $\mathbb{l}$. The standardized protocol consisted in three 3-minute phases of resting (lying down, standing up and sitting) and 3-minute walking/running phases (from $0.5 \mathrm{~m} / \mathrm{s}$ to exhaustion; with steps of $0.5 \mathrm{~m} / \mathrm{s}$ ). For each subject in this database, an ECG segment with a duration of at least least six minutes was chosen and annotated by experts for performance evaluation.

TABLE I

ANTHROPOMETRIC PARAMETERS OF THE SUBJECTS IN THE WEARABLE TECHNOLOGY DATABASE.

\begin{tabular}{|c|c|c|}
\hline Characteristic & mean $\pm \operatorname{std}(N=13)$ & Range \\
\hline \hline Age [years] & $35.95 \pm 6.74$ & $27-46$ \\
\hline Height $[\mathrm{m}]$ & $1.82 \pm 0.07$ & $1.72-1.95$ \\
\hline Weight $[\mathrm{kg}]$ & $75.88 \pm 6.35$ & $65-87$ \\
\hline
\end{tabular}

\section{RESUlts AND DisCUSSION}

As the core of all MM operations, the shape and length of the SE should be carefully chosen as they play important roles in the outcome of these operators [प][8][10]. For instance, the average of an opening and closing of a signal with a small length flat SE, e.g. a $6 \mathrm{~ms}$ zero vector, can be used for noise suppression [7] while the same average with a peaky SE tends to enhance peaks and valleys in the signal and discard other activities.

In ECG waveform detection, specifically QRS complex detection, MM approaches use fixed-shape and -magnitude QRS-like SE to manipulate the signal and detect the R-waves. However, the magnitude and length of the SE are important factors. The steeper the slope, the less sensitive the operator becomes to low amplitude activities in the signal. Therefore, when perturbations are present in a part of a signal, SEs with steeper slopes tend to detect more dominant changes, resulting in a better feature signal. However, if the magnitude is too large, sometimes the QRS complexes may not be detected. This phenomenon can be observed when arrhythmia is present. The length of the SE is decisive as well. A longer SE has more averaging effect, and therefore removes more unwanted activities. However, this noise reduction significantly decreases the amplitude of the R-waves. Also, it changes the relative positions of other fiducial points, especially the onsets and offsets. For this reason, a compromise should be made in order to have a more efficient detection. 


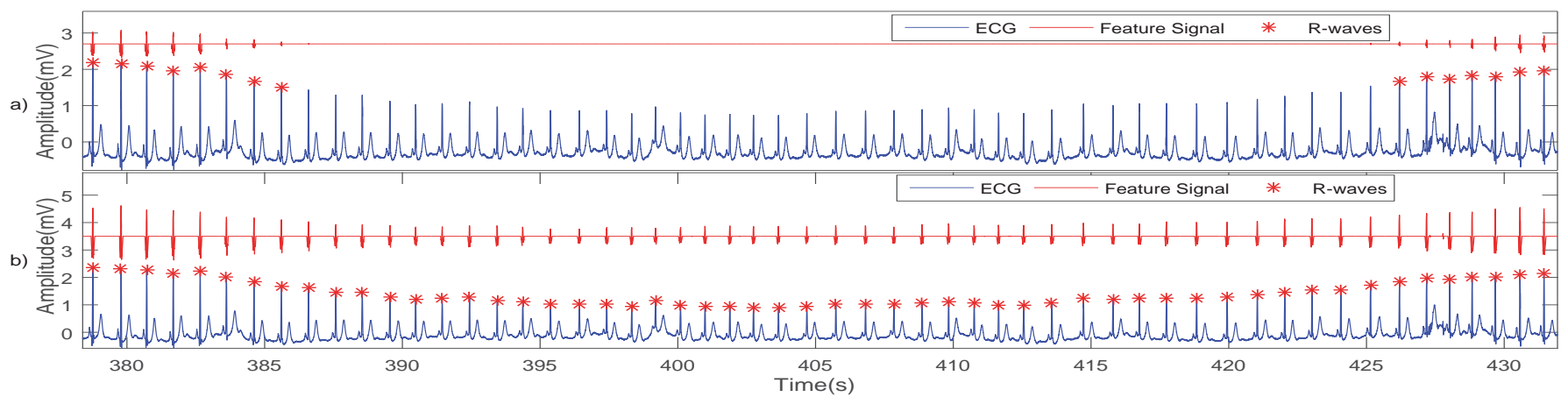

Fig. 6. a) Illustration of MM filtering on tape 106 from the MIT/BIH arrhythmia database using a fixed SE throughout the ECG. The used SE has a magnitude equal to that of the first peak in this figure. A DC value of $2.7 \mathrm{mv}$ is added to the feature signal. b) MM filtering output using an adaptive SE. A DC value of $3.5 \mathrm{mv}$ is aded to the feature signal.

Our observations have led us to the conclusion that a fixed SE may not always detect QRS complexes, especially when sudden changes, amplitude modulation, or baseline drift are present in the ECG. Fig. 6-a illustrates the fixed SE problem. In this example, the SE is simply too large in magnitude to detect all heartbeats. As a result, low amplitude heartbeats are considered as undesired ECG activities. Once the heartbeats get larger again at the end of the signal, they are correctly detected. Fig. 6-b illustrates the outcome of an adaptive SE on the same ECG segment, where these beats are correctly detected. In order to detect the low amplitude heartbeats, one can use an SE with a smaller magnitude. However, the output of the MM filtering phase will become more sensitive to smaller changes in the ECG and the feature signal will have active periods where there is no heartbeat.

As the heart of AMM, the SE is adapted on a beat-to-beat basis. This adaptation changes the locations of the fiducial points as well as their amplitudes in the SE. The updated SE enables AMM to extract the other fiducial points more accurately. As a result, the outcome of the MM filter is less affected by sudden changes compared to a fixed SE.

\subsection{Finding Optimized Parameters}

In order to optimize performance, we studied the effect of magnitude and length of the SE on AMM performance. To this end, true positives (TP), false negative (FN), and false positives (FP) values were studied. TP indicates the number of correctly detected QRS complexes while FN represents the number of miss-detected complexes. FP specifies the number of complexes declared by AMM where no actual heartbeat takes place in the ECG. Using these statistics, the detection error rate (DER) as well as sensitivity (Se), and positive prediction value (PPV) were computed through Eq. 12-14. In order to find the optimal parameters for AMM, these measures were calculated over all 48 tapes of the MIT/BIH arrhythmia database.

$$
\begin{gathered}
\text { Sensitivity }(S e)=\frac{T P}{T P+F N} \\
\text { PositivePredictionValue }(P P V)=\frac{T P}{T P+F P} \\
\text { Detection Error Rate }(D E R)=\frac{F P+F N}{\text { Total No. of Beats }}
\end{gathered}
$$

In figures $\square$ and $\mathbb{Q}$, we use DetectionAccuracy defined as 1 - DetectionError for demonstration purposes. Fig. ㅁ-a shows the effect of length of the SE on the overall QRS complex detection performance of AMM. The update phase of AMM was modified to reconstruct the SEs with a fraction of the length of the extracted complexes. We studied different lengths in a range of 0.6 to 1.3 times the extracted length of QRS complexes, by steps of 0.1. Results show consistently high performance in the range of 0.7 to 1.1, with the actual QRS complex length as the optimal parameter. However the performance drops as longer SEs are reconstructed.

In Fig $\mathbb{Z}-\mathrm{b}$, we study how the magnitude of the SE affects R-wave detection performance. In order to find the best magnitude, the SE was scaled from 0.05 up to 1.15 times the actual magnitude of the QRS complex with a step size of 0.05 . The performance strictly improves from the beginning to 0.6 . After this point, the performance gradually decreases until 0.95 , and then a drastic drop in performance is observed as the magnitude of the SE gets larger than the actual complex. This sudden drop in performance is introduced by the increasing number of undetected heartbeats, which have lower amplitudes than their predecessors. This phenomenon can be the consequence of respiratory modulation of the ECG or a simple baseline drift.

Heartbeats in the MIT/BIH database are annotated with different labels representing normal and abnormal beats, e.g. N represents normal beats and V is the label of premature ventricular beats. In order to find the optimal thresholds for Eq. 11, the PeakActivity feature (Eq. 10) was calculated for normal and ventricular beats, namely premature ventricular contractions, 


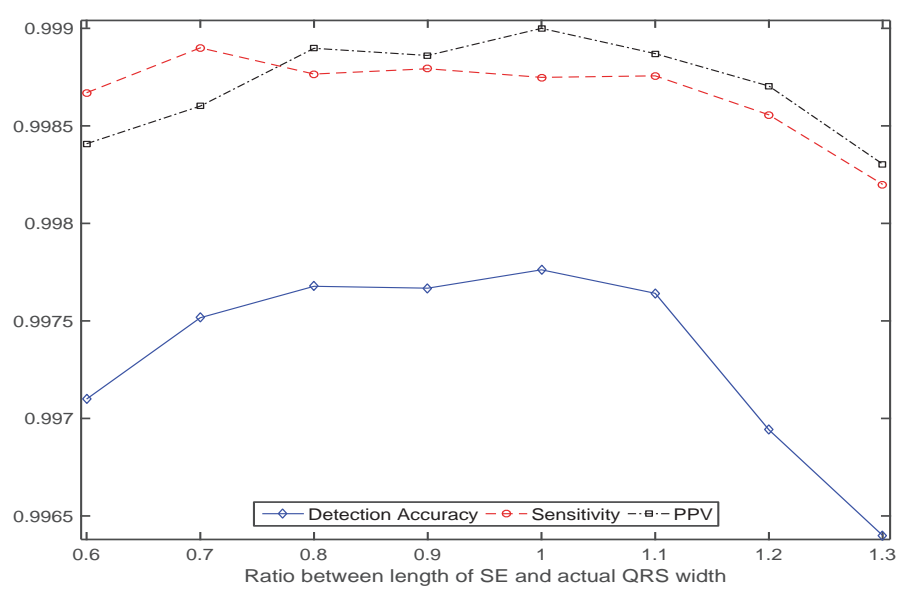

(a)

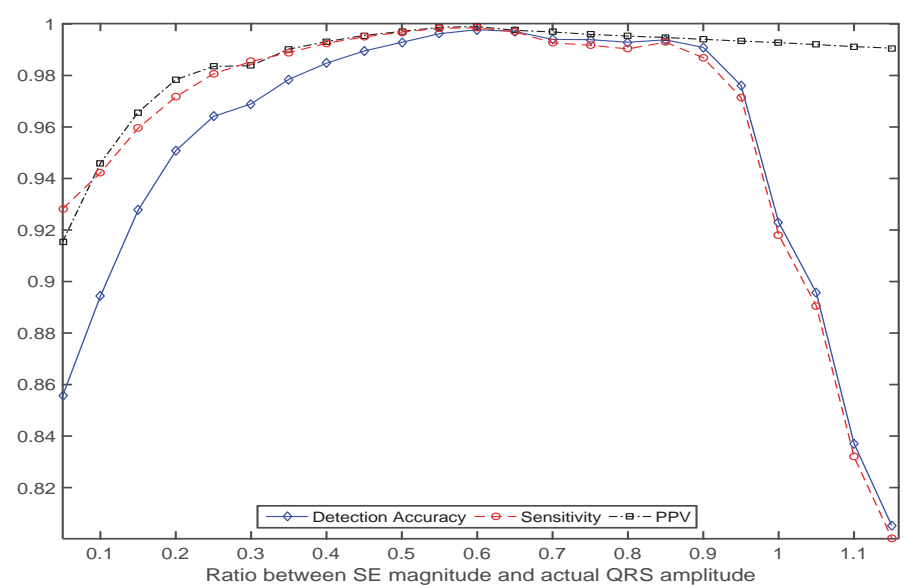

(b)

Fig. 7. (a) Evolution of the detection accuracy (1-DER), sensitivity and PPV with respect to the ratio between the length of the SE and that of the actual QRS complex. (b) Same evolution with respect to the ratio between the magnitude of the SE and that of the actual QRS complex.

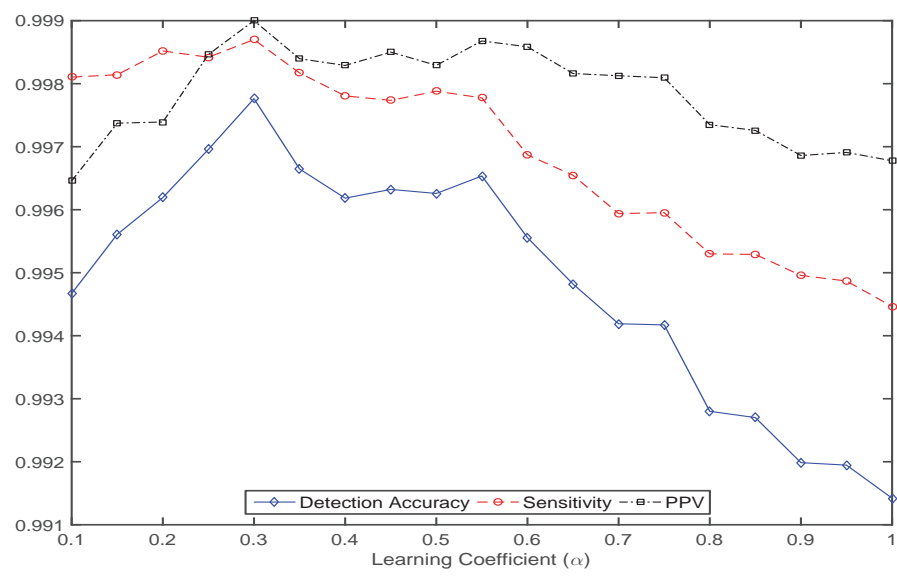

(a)

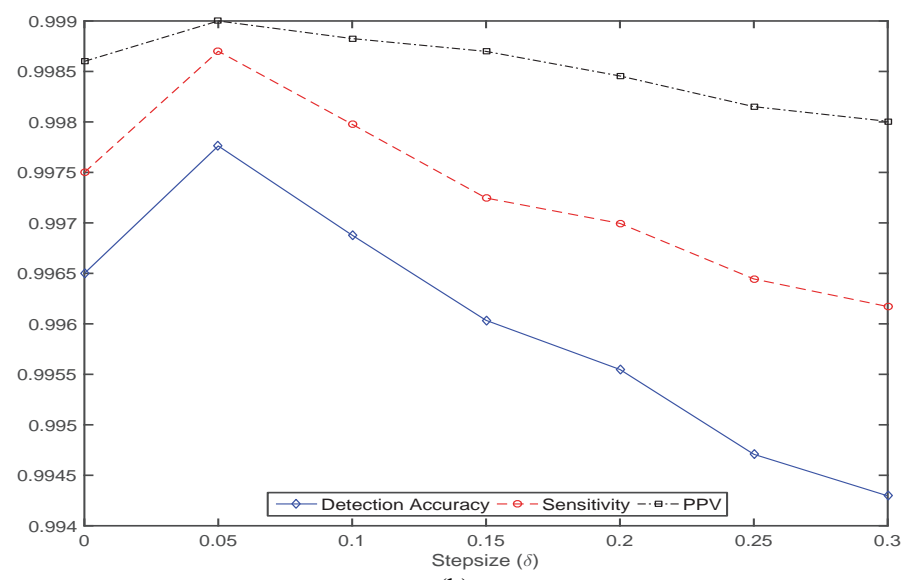

(b)

Fig. 8. (a) Evolution of the detection accuracy (1-DER), sensitivity and PPV with respect to the learning coefficient ( $\alpha$ ) in Eq. $\mathbf{8}$ and $\mathbf{9}$. (b) Same evolution with respect to different stepsizes $(\delta)$. Stepsize $(\delta)$ fine tunes the learning coefficient, as described in Eq. II.

supraventricular premature beats. By comparing the PeakActivity feature of these two groups, we found that there is a difference of at least $10 \%$ in PeakActivity value between these two sets of beats. Therefore, $t h_{l o w}$ and $t h_{h i g h}$ were selected respectively as 0.9 and 1.1 . Using the optimal length, magnitude, and PeakActivity thresholds, we studied the effect of the learning coefficient on AMM performance. As shown in Fig. 8-a, the optimal learning coefficient, i.e. $\alpha$ in Eq. W, was 0.3. Fig. 8-b illustrates how fine tuning the learning coefficient enhances the overall performance of AMM. Updating the learning coefficient based on the extracted PeakActivity feature is especially important when abnormal beats with extra large amplitude take place in the ECG. If the learning coefficient is not updated, extra large (or small) outlier beats can alter the SE excessively, which results in a feature signal that is less (more) sensitive to changes in the ECG.

\subsection{AMM Performance Evaluation}

Over the past two decades, many QRS detection approaches have been proposed. Therefore, in order to compare AMM performance with the state-of-the-art, we used the MIT/BIH arrhythmia database. Figure 9-a illustrates AMM performance when strong baseline drift and muscle activity pollute the ECG. In this example, one can see a low-amplitude active period in the feature signal due to the elevated T-wave right after the first QRS complex. Fig. 9-b displays how AMM performs when switches of QRS polarity take place. This part of the tape 200 is especially selected, not only to show how AMM performs when premature ventricular contractions are present, but also to show the benefit of adapting the learning coefficient. As one can see, the QRS complex in the 110-111 second period has a significantly larger PeakActivity in the feature signal compared to that of the previous beat. Subsequently, the learning coefficient is decreased through Eq. 11, and the SE is less affected in the update phase of the algorithm. This leads to an active period with a duration longer than $70 \mathrm{~ms}$ and therefore the detection of the final QRS complex in this figure. 


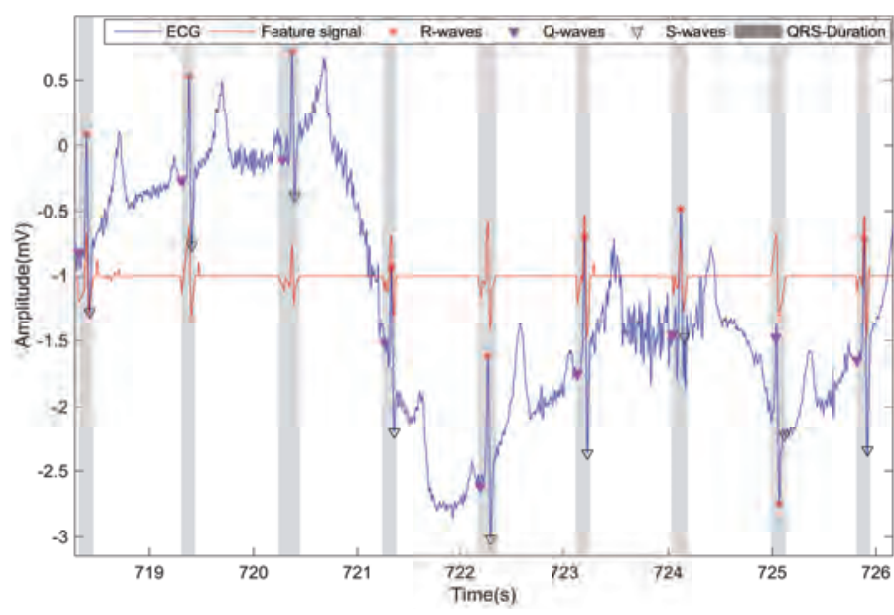

(a)

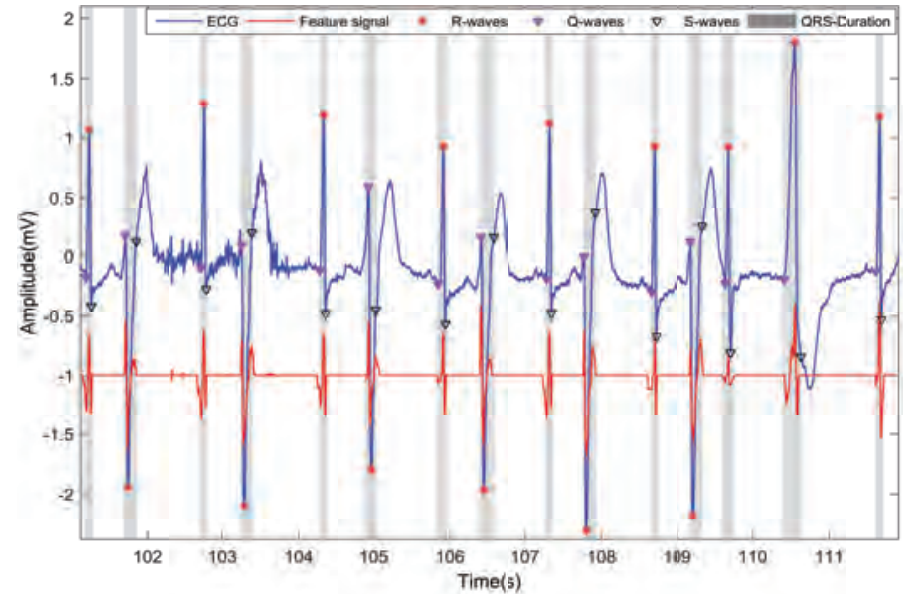

(b)

Fig. 9. (a) Illustration of AMM performance on tape no. 117 from the MIT/BIH arrhythmia. A DC value of $-1 \mathrm{mv}$ is added to the feature signal.(b) AMM performance on tape no. 200 from the MIT/BIH arrhythmia. A DC value of $-1 \mathrm{mv}$ is added to the feature signal.

When it comes to Q- and S-points, the presence of baseline and muscle activities, e.g. Q-a, lowers the estimation accuracy. Due to the fact that these waves do not necessarily form a valley in the ECG. However, our observations have shown that, even in the presence of these perturbations, fiducial point extraction is acceptable.

More detailed evaluation on tapes from the MIT/BIH database can be found in Table $\mathbb{U l}$. Note that what is performed is QRS detection not R-peak extraction. Consequently, a detection is considered valid whenever the annotated R-peak is inside the detected QRS complex. For each tape, numbers of false positives and false negatives and total number of beats as well as sensitivity, positive prediction value and detection error rate are reported. Furthermore, Table Wl compares the performance of AMM to those of well-known QRS detection methods. As shown in this table, AMM provides better or comparable results.

To the best of our knowledge, in the state-of-the-art, most methods isolate the location of QRS complexes by means of thresholding and then extract the R-peaks using local search [5][6][8][24]. However, AMM not only provides the necessary means to extract the R-peaks, but also the onset, offset, Q- and S-points can be estimated directly from the feature signal. Location and amplitude of these fiducial points can be used in beat classification and arrhythmia analysis [26][27][28]. In [26], features based on RR intervals, QRS duration and segmented morphology ( down sampled extracted QRS complex) were used to develop an automated heartbeat classifier. Arrhythmia analysis is possible through the processing of RR-intervals [29] using adaptive filters [30] or time-frequency analysis [3T]. Nevertheless, once accurate locations of QRS fiducial points are available, heartbeat classification and arrhythmia detection can be improved [26]. In this context we analyzed the performance of AMM on the manually annotated QRS complexes in the QT database, with results presented in Table $\mathbb{V}$. The QRS complex annotation provided in this database consists of the locations of R-peaks as well as the onset and offset points. In order to further assess AMM performance on Q- and S-point extraction, the ground truth for these fiducial points were respectively considered as the maximum (minimum) between the onset-peak and the peak-offset interval for a positive (negative) beat.

In table $\mathbb{L V}$, the sensitivity and detection rate for each fiducial point in the QRS complex is reported. For each fiducial point an error tolerance was considered in order to measure the overall AMM performance on each fiducial point. Considering the sampling frequency of the records available in the QT database, i.e. $250 \mathrm{~Hz}$, respectively one and three samples were considered as tolerable error for the Q-/R-/S- and QRS-onset/QRS-offset points. Fig. W0 shows a box plot of the detection error rate for each fiducial point. Furthermore, we computed AMM delineation errors with respect to manual annotations that was provided by a cardiologist for the QT database. Table $\nabla$ reports these values for AMM and compares them with the work of Martinez et al. [18]. In tables $\mathbb{Z}$ and $\nabla$, reported results are in comparison with the label provided by the cardiologist who has annotated all records in the QTDB.

Another contribution of the proposed algorithm is the ability to be used in body-area network platforms. While having limited resources, these platforms are expected to offer long term monitoring of subjects and provide robust extraction of heartbeats as a first step to more elaborated algorithms such as arrhythmia detection and heart rate variability analysis. The computation load of AMM consists of two elements namely, MM operation and peak detection, and SE update. The total computation load can be expressed as $O(l \times n)+O(l \times r)$ where $n$ represents the number of samples in the ECG, $l$ the length of the structuring element and $r$ the number of detected heartbeats. Since $l \ll n$ and $r \ll n$ the second term can be omitted and the order of complexity of AMM can be written as $O(n)$.

AMM performs well on the MIT/BIH database. However, ECGs in the aforementioned databases are recorded by standard acquisition devices and may not represent the obstacles of portable and continuous recordings such as electrode movement and contact problems, continuous muscle activity contamination and presence of large baseline drift. Therefore, AMM performance 
TABLE II

PERFORMANCE OF AMM ON QRS COMPLEX DETECTION ON MIT/BIH ARRHYTHMIA DATABASE.

\begin{tabular}{|c|c|c|c|c|c|c|}
\hline Tape & Beats & FP & $\mathrm{FN}$ & QRS DER \% & Sensitivity & PPV \\
\hline 100 & 2273 & 0 & 0 & 0 & 1 & 1 \\
\hline 101 & 1865 & 1 & 0 & 0.0536 & 1 & 0.9995 \\
\hline 102 & 2187 & 0 & 0 & 0 & 1 & 1 \\
\hline 103 & 2084 & 0 & 0 & 0 & 1 & 1 \\
\hline 104 & 2229 & 7 & 1 & 0.3589 & 0.9996 & 0.9969 \\
\hline 105 & 2572 & 22 & 15 & 1.4386 & 0.9942 & 0.9915 \\
\hline 106 & 2027 & 0 & 4 & 0.1973 & 0.998 & 1 \\
\hline 107 & 2137 & 0 & 1 & 0.0468 & 0.9995 & 1 \\
\hline 108 & 1763 & 3 & 17 & 1.1344 & 0.9904 & 0.9983 \\
\hline 109 & 2532 & 0 & 1 & 0.0395 & 0.9996 & 1 \\
\hline 111 & 2124 & 2 & 1 & 0.1412 & 0.9995 & 0.9991 \\
\hline 112 & 2539 & 0 & 0 & 0 & 1 & 1 \\
\hline 113 & 1795 & 0 & 0 & 0 & 1 & 1 \\
\hline 114 & 1879 & 4 & 2 & 0.3193 & 0.9989 & 0.9979 \\
\hline 115 & 1953 & 0 & 0 & 0 & 1 & 1 \\
\hline 116 & 2412 & 0 & 20 & 0.8292 & 0.9917 & 1 \\
\hline 117 & 1535 & 0 & 0 & 0 & 1 & 1 \\
\hline 118 & 2278 & 2 & 0 & 0.0878 & 1 & 0.9991 \\
\hline 119 & 1987 & 0 & 0 & 0 & 1 & 1 \\
\hline 121 & 1863 & 0 & 2 & 0.1074 & 0.9989 & 1 \\
\hline 122 & 2476 & 0 & 0 & 0 & 1 & 1 \\
\hline 123 & 1518 & 0 & 0 & 0 & 1 & 1 \\
\hline 124 & 1619 & 0 & 0 & 0 & 1 & 1 \\
\hline 200 & 2601 & 4 & 1 & 0.1922 & 0.9996 & 0.9985 \\
\hline 201 & 1963 & 0 & 12 & 0.6113 & 0.9939 & 1 \\
\hline 202 & 2136 & 0 & 2 & 0.0936 & 0.9991 & 1 \\
\hline 203 & 2980 & 18 & 12 & 1.0067 & 0.996 & 0.994 \\
\hline 205 & 2656 & 1 & 2 & 0.113 & 0.9992 & 0.9996 \\
\hline 207 & 1860 & 3 & 3 & 0.3226 & 0.9984 & 0.9984 \\
\hline 208 & 2955 & 2 & 9 & 0.3723 & 0.997 & 0.9993 \\
\hline 209 & 3005 & 4 & 0 & 0.1331 & 1 & 0.9987 \\
\hline 210 & 2650 & 3 & 4 & 0.2642 & 0.9985 & 0.9989 \\
\hline 212 & 2748 & 0 & 0 & 0 & 1 & 1 \\
\hline 213 & 3251 & 0 & 3 & 0.0923 & 0.9991 & 1 \\
\hline 214 & 2262 & 0 & 0 & 0 & 1 & 1 \\
\hline 215 & 3363 & 0 & 0 & 0 & 1 & 1 \\
\hline 217 & 2208 & 6 & 4 & 0.4529 & 0.9982 & 0.9973 \\
\hline 219 & 2154 & 0 & 0 & 0 & 1 & 1 \\
\hline 220 & 2048 & 0 & 0 & 0 & 1 & 1 \\
\hline 221 & 2427 & 0 & 6 & 0.2472 & 0.9975 & 1 \\
\hline 222 & 2483 & 4 & 4 & 0.3222 & 0.9984 & 0.9984 \\
\hline 223 & 2605 & 2 & 0 & 0.0768 & 1 & 0.9992 \\
\hline 228 & 2053 & 11 & 8 & 0.9255 & 0.9961 & 0.9946 \\
\hline 230 & 2256 & 0 & 0 & 0 & 1 & 1 \\
\hline 231 & 1571 & 0 & 2 & 0.1273 & 0.9987 & 1 \\
\hline 232 & 1780 & 4 & 0 & 0.2247 & 1 & 0.9978 \\
\hline 233 & 3079 & 5 & 1 & 0.1949 & 0.9997 & 0.9984 \\
\hline 234 & 2753 & 0 & 0 & 0 & 1 & 1 \\
\hline Total & 109494 & 108 & 137 & 0.2238 & 0.9987 & 0.999 \\
\hline
\end{tabular}

TABLE III

COMPARISON OF PERFORMANCE WITH PREVIOUSLY PROPOSED METHODS ON MIT/BIH ARRHYTHMIA DATABASE.

\begin{tabular}{|c|c|c|c|c|c|}
\hline Method & No. of Beats & FP & FN & DER \% & Ref. No. \\
\hline AMM (this work) & 109494 & 108 & 137 & $\overline{0.224}$ & - \\
\hline Pan and Tompkins & 109809 & 507 & 277 & 0.710 & [5] \\
\hline Li et al. & 104184 & 65 & 112 & 0.170 & [6] \\
\hline Zhang and Lian & 109510 & 204 & 213 & 0.38 & [8] \\
\hline Ravanshad et al. & 109428 & 651 & 1216 & 1.71 & [I7] \\
\hline Martinez et al. & 109428 & 153 & 220 & 0.34 & [18] \\
\hline Bahoura et al. & 109809 & 135 & 184 & 0.29 & [II9] \\
\hline Moody and Mark & 109428 & 94 & 1861 & 1.79 & [20] \\
\hline Lee et al. & 109481 & 137 & 135 & 0.43 & [21] \\
\hline Hamilton and Tompkins & 109267 & 248 & 340 & 0.54 & [22] \\
\hline Poli et al. & 109963 & 545 & 441 & 0.90 & [23] \\
\hline Chen et al. & 102654 & 529 & 459 & 0.96 & [24] \\
\hline Afonso et al. & 90909 & 406 & 374 & 0.86 & [2.5] \\
\hline
\end{tabular}


TABLE IV

AMM PERFORMANCE ON THE MANUALLY ANNOTATED BEATS FOR QRS FIDUCIAL POINTS, PHYSIONET QT DATABASE.

\begin{tabular}{|c|c|c|c|c|}
\hline Fiducial point & Sensitivity & Detection Rate & Tolerance $(\mathrm{ms})$ & DER\% $(\mathrm{mean} \pm$ std) \\
\hline \hline R-peak & 0.9987 & 0.9990 & 4 & $0.001 \pm 0.0011$ \\
\hline QRS-Onset & 0.9684 & 0.9791 & 12 & $0.0209 \pm 0.0266$ \\
\hline Q-point & 0.9903 & 0.9902 & 4 & $0.0098 \pm 0.0201$ \\
\hline S-point & 0.9966 & 0.9966 & 4 & $0.0036 \pm 0.04$ \\
\hline QRS-Offset & 0.9820 & 0.9818 & 12 & $0.0182 \pm 0.0055$ \\
\hline
\end{tabular}

TABLE V

AMM Delineation Performance AND Comparison, QT Database.

\begin{tabular}{|c|c|c|c|c|}
\hline Method & $\begin{array}{c}Q R S_{\text {Onset }} \\
\text { mean } \pm \text { std }(\mathrm{ms})\end{array}$ & $\begin{array}{c}R-\text { waves } \\
\text { mean } \pm \text { std }(\mathrm{ms})\end{array}$ & $\begin{array}{c}Q R S_{O f f s e t} \\
\text { mean } \pm \text { std }(\mathrm{ms})\end{array}$ & Reference \\
\hline \hline AMM & $6.1 \pm 8.3$ & $2.1 \pm 1.2$ & $1.5 \pm 4.2$ & - \\
\hline Martinez et al. & $4.6 \pm 7.7$ & Not Reported & $0.8 \pm 8.7$ & {$[\overline{[18}]$} \\
\hline
\end{tabular}

was evaluated on a dataset of ECGs that were recorded using smart shirts. In this database, subjects were asked to perform a series of walking/running exercises. The levels of exercises were categorized into low, moderate, and vigorous classes. For each subject, a six minute vigorous ECG segment was chosen and annotated by experts in order to evaluate the performance of AMM in extreme conditions. Figure 1 illustrates AMM outcome on one of these challenging segments. In this figure, the ECG suffers from a large baseline drift that is contaminating the signal, making QRS extraction a hard task.

The over all performance of AMM was compared and reported in table VI, against two other methods namely, Pan-Tompkins and fixed mathematical morphology. Results show how AMM outperforms these methods and highlights the importance of the adaptation of the structuring element. Overall, a sensible improvement in performance with over ten and five percent was achieved respectively in comparison to Pan-Tompkins and fixed mathematical morphology. This performance together with the low computation cost of AMM, makes it a suitable approach for body area network platforms, in which power consumption plays a vital role.

The adaptation performed on the structuring element, changes the behavior of MM filtering phase. During the initialization of the algorithm, the structuring element is adapted so that it represents the general QRS complex morphology of the subject. As AMM processes more QRS complexes, the adaptation on the structuring element makes AMM more robust against possible noise in the ECG. However, our observation on the miss detected complexes show that AMM under senses when beats that become too wide (longer duration compared to previous beats) and at the same time lose their peaky shape. Moreover, false positives by AMM are mostly due to impulsive baseline changes, isolated QRS-like artifacts and clipping of the signal.

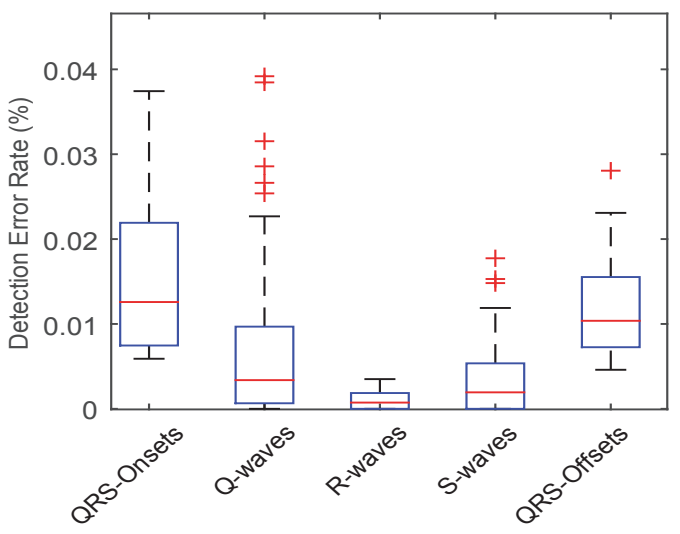

Fig. 10. Illustration of AMM performance on the QT database for each fiducial point.

TABLE VI

COMPARISON OF AMM PERFORMANCE WITH OTHER METHODS ON THE WEARABLE ECG DATABASE.

\begin{tabular}{|c|c|c|c|c|c|c|c|}
\hline Method & No. of Beats & FP & FN & Detection Rate \% & Sensitivity & PPV \% & Ref. No. \\
\hline \hline AMM & 11806 & 59 & 142 & 98.39 & 0.9883 & 0.995 & - \\
\hline Fixed-MM & 11806 & 161 & 780 & 92.57 & 0.9362 & 0.9836 & - \\
\hline Pan-Tompkins & 11806 & 534 & 1085 & 88.33 & 0.912 & 0.9511 & {$[$ []] } \\
\hline
\end{tabular}




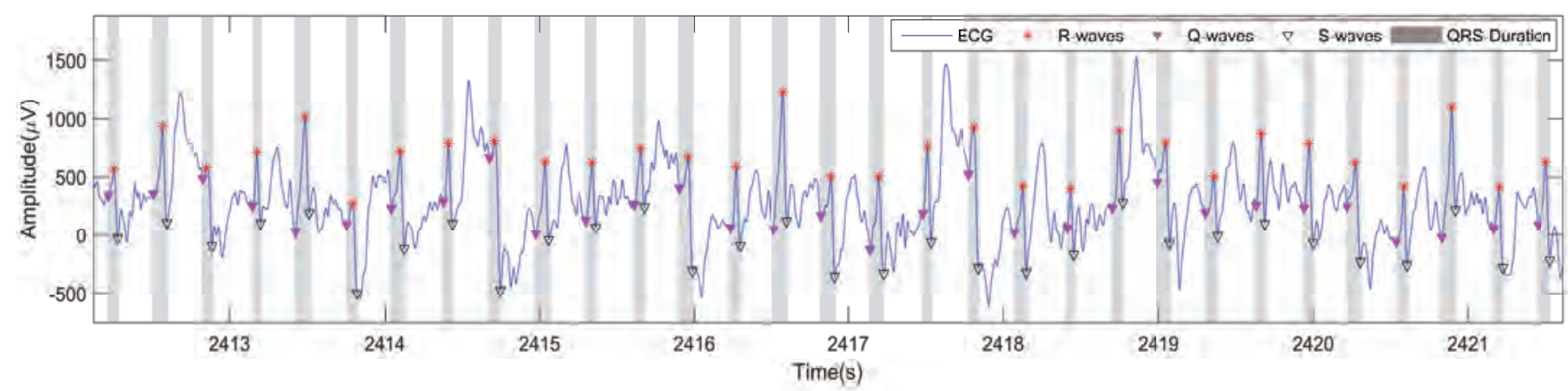

Fig. 11. Illustration of AMM performance on an ECG recored by a smart shirt, while subject is performing a vigorous running activity.

\section{CONCLUSION}

In this paper we present an adaptive mathematical morphology scheme for QRS complex and fiducial point detection. The proposed approach updates the structuring element used in MM operators after the detection of each heartbeat for a better and more reliable detection. The adaptation makes the algorithm robust against perturbations such as baseline drifts and muscle activity interferences. Achieving a high detection rate of $98.39 \%$ and a sensitivity of 0.988 against a smart shirt database, while benefiting from low computation load, makes this approach a suitable choice where limited resources are available such as for body-area networks.

Despite of the term "QRS detectors", most methods focus on detecting the R-waves and not the other fiducial points in the QRS complex such as QRS-onset, QRS-offset, Q-point and S-point. The proposed method detects the aforementioned fiducial points alongside the R-peaks with a low detection error rate. These fiducial points provide the necessary information for more precise arrhythmia detection and heartbeat classification. Against the Physionet QT database, AMM achieved a detection rate of $99.90 \%$ for the R-peaks while estimating the location of the QRS-onsets and QRS-offsets respectively with detection rates of $97.91 \%, 98.18 \%$.

\section{ACKNOWLEDGEMENTS}

The authors would like to thank all CSEM collaborators who were involved in the design and development of the thoraxlocated devices, those involved in the embedded signal processing as well as those in charge of the measurement campaign.

This study was performed in the framework of the Nano-Tera initiative supported by the Swiss National Science Foundation (SNSF), [SwissNano-Tera RTD ObeSense].

\section{REFERENCES}

[1] R. M. Rangayyan, Biomedical signal analysis. IEEE press New York, 2002.

[2] T. F. of the European Society of Cardiology, T. F. of the European Society of Cardiology et al., "the north american society of pacing and electrophysiology. heart rate variability: standards of measurement, physiological interpretation and clinical use," Journal of Circulation, vol. 93, no. 5, pp. 1043-1065, 1996.

[3] B.-U. Kohler, C. Hennig, and R. Orglmeister, "The principles of software qrs detection," IEEE Magazine of Engineering in Medicine and Biology, no. 1, pp. 42-57, 2002.

[4] G. M. Friesen, T. C. Jannett, M. A. Jadallah, S. L. Yates, S. R. Quint, and H. T. Nagle, "A comparison of the noise sensitivity of nine qrs detection algorithms," IEEE Transactions on Biomedical Engineering, no. 1, pp. 85-98, 1990.

[5] J. Pan and W. J. Tompkins, "A real-time qrs detection algorithm," IEEE Transactions on Biomedical Engineering, no. 3, pp. 230-236, 1985.

[6] C. Li, C. Zheng, and C. Tai, "Detection of ecg characteristic points using wavelet transforms," IEEE Transactions on Biomedical Engineering, no. 1, pp. 21-28, 1995.

[7] P. Trahanias, "An approach to qrs complex detection using mathematical morphology," IEEE Transactions on Biomedical Engineering, no. 2, pp. 201-205, 1993.

[8] F. Zhang and Y. Lian, "Qrs detection based on multiscale mathematical morphology for wearable ecg devices in body area networks," IEEE Transactions on Biomedical Circuits and Systems, no. 4, pp. 220-228, 2009.

[9] S. Yazdani and J.-M. Vesin, "Adaptive mathematical morphology for qrs fiducial points detection in the ecg," in The 41st annual international conference of Computing in Cardiology, 2014, pp. 725-728.

[10] P. Maragos and R. W. Schafer, "Morphological systems for multidimensional signal processing," Proceedings of the IEEE, no. 4, pp. 690-710, 1990.

[11] B. Surawicz, R. Childers, B. J. Deal, and L. S. Gettes, "Recommendations for the standardization and interpretation of the electrocardiogram: Part iii: Intraventricular conduction disturbances: A scientific statement from the american heart association electrocardiography and arrhythmias committee, council on clinical cardiology," Journal of Circulation, vol. 119, no. 10, pp. 235-240, 2009.

[12] J. M. Arribas Leal, D. A. Pascual-Figal, M. A. Vidal, F. M. Ortuño, F. G. García, J. G.-P. del Corral, J. A. Ruipérez Abizanda, G. T. Martínez, M. V. Chávarri, and R. A. Meca, "Qrs duration and early hemodynamic instability after coronary revascularization surgery," Journal of Revista Española de Cardiología (English Edition), no. 6, pp. 652-659, 2009.

[13] C. Wolpert, C. Veltmann, R. Schimpf, C. Antzelevitch, I. Gussak, and M. Borggrefe, "Is a narrow and tall qrs complex an ecg marker for sudden death?" Journal of Heart Rhythm, vol. 5, no. 9, pp. 1339-1345, 2008.

[14] A. D. Sharma, R. Yee, G. Guiraudon, and G. J. Klein, "Sensitivity and specificity of invasive and noninvasive testing for risk of sudden death in wolff-parkinson-white syndrome," Journal of the American College of Cardiology, vol. 10, no. 2, pp. 373-381, 1987.

[15] G. B. Moody and R. G. Mark, "The impact of the mit-bih arrhythmia database," IEEE Magazine of Engineering in Medicine and Biology, vol. 20, no. 3, pp. 45-50, 2001. 
[16] P. Laguna, R. Mark, A. Goldberg, and G. Moody, "A database for evaluation of algorithms for measurement of qt and other waveform intervals in the ecg," in In Proceedings of Computers in Cardiology, 1997, pp. 673-676.

[17] N. Ravanshad, H. Rezaee-Dehsorkh, R. Lotfi, and Y. Lian, "A level-crossing based qrs-detection algorithm for wearable ecg sensors," IEEE Journal of Biomedical and Health Informatics, vol. 18, no. 1, pp. 183-192, 2014.

[18] J. P. Martínez, R. Almeida, S. Olmos, A. P. Rocha, and P. Laguna, "A wavelet-based ecg delineator: evaluation on standard databases," IEEE Transactions on Biomedical Engineering, vol. 51, no. 4, pp. 570-581, 2004.

[19] M. Bahoura, M. Hassani, and M. Hubin, "Dsp implementation of wavelet transform for real time ecg wave forms detection and heart rate analysis," Computer methods and programs in biomedicine, vol. 52, no. 1, pp. 35-44, 1997.

[20] G. B. Moody and R. G. Mark, "Development and evaluation of a 2-lead ecg analysis program," Computers in cardiology, vol. 9, pp. 39-44, 1982.

[21] J. Lee, K. Jeong, J. Yoon, and M. Lee, "A simple real-time qrs detection algorithm," in Proceedings of the 18th Annual International Conference of the IEEE, vol. 4, 1996, pp. 1396-1398.

[22] P. S. Hamilton and W. J. Tompkins, "Quantitative investigation of qrs detection rules using the mit/bih arrhythmia database," IEEE Transactions on Biomedical Engineering, vol. BME-33, no. 12, pp. 1157-1165, 1986.

[23] R. Poli, S. Cagnoni, and G. Valli, "Genetic design of optimum linear and nonlinear qrs detectors," IEEE Transactions on Biomedical Engineering, vol. 42, no. 11, pp. 1137-1141, 1995.

[24] S.-W. Chen, H.-C. Chen, and H.-L. Chan, "A real-time qrs detection method based on moving-averaging incorporating with wavelet denoising," Computer Methods and Programs in Biomedicine, vol. 82, no. 3, pp. 187-195, 2006.

[25] V. Afonso, W. J. Tompkins, T. Nguyen, and S. Luo, "Ecg beat detection using filter banks," IEEE Transactions on Biomedical Engineering, vol. 46, no. 2, pp. 192-202, 1999.

[26] P. De Chazal, M. O'Dwyer, and R. B. Reilly, "Automatic classification of heartbeats using ecg morphology and heartbeat interval features," IEEE Transactions on Biomedical Engineering, no. 7, pp. 1196-1206, 2004.

[27] I. Christov, I. Jekova, and G. Bortolan, "Premature ventricular contraction classification by the k th nearest-neighbours rule," Journal of Physiological Measurement, vol. 26, no. 1, pp. 123-130, 2005.

[28] P. de Chazal and R. Reilly, "A patient-adapting heartbeat classifier using ecg morphology and heartbeat interval features," IEEE Transactions on Biomedical Engineering, vol. 53, no. 12, pp. 2535-2543, 2006.

[29] M. G. Tsipouras, D. I. Fotiadis, and D. Sideris, "An arrhythmia classification system based on the rr-interval signal," Artificial Intelligence in Medicine, vol. 33, no. 3, pp. 237-250, 2005.

[30] N. V. Thakor and Y.-S. Zhu, "Applications of adaptive filtering to ecg analysis: noise cancellation and arrhythmia detection," IEEE Transactions on Biomedical Engineering, vol. 38, no. 8, pp. 785-794, 1991.

[31] V. X. Afonso and W. J. Tompkins, "Detecting ventricular fibrillation," IEEE Journal of Engineering in Medicine and Biology Magazine, no. 2, pp. $152-159,1995$.

Sasan Yazdani received his M.Sc. in artificial intelligence and robotics from Iran University of Science and Technology (IUST), in 2013. He is currently working towards his Ph.D. degree in biomedical signal processing at Swiss Federal Institute of Technology, Lausanne, Switzerland (EPFL). His professional research interests are biomedical signal processing, adaptive signal analysis and machine learning.

Jean-Marc Vesin graduated from the Ecole Nationale Supérieure d'Ingénieurs Electriciens de Grenoble (ENSIEG, Grenoble, France) in 1980. He received his M.Sc. from Laval University, Québec city, Canada, in 1984. After two years in the industry, he joined the Swiss Federal Institute of Technology, Lausanne, Switzerland (EPFL), where he obtained his Ph.D. in 1992. He currently heads the Applied Signal Processing Group (ASPG) at EPFL. His main interests are biomedical signal processing, adaptive signal analysis, nonlinear signal modeling and analysis. He has authored or co-authored more than sixty publications in peer-reviewed journals, as well as several book chapters. 